\title{
Bioinformatics analysis of prognostic value and prospective pathway signal of miR-30a in ovarian cancer
}

\author{
Weijia $\mathrm{Lu}^{1 \dagger}$, Yunyu $\mathrm{Wu}^{2 \dagger}$, Can Xiong $\mathrm{Lu}^{3}$, Ting Zhu ${ }^{4}$, Zhong Lu Ren ${ }^{5}$ and Zhiwu Yü ${ }^{4^{*}}$
}

\begin{abstract}
Objective: MicroRNAs (MiRNAs) is thought to play a critical role in the initiation and progress of ovarian cancer (OC). Although miRNAs has been widely recognized in ovarian cancer, the role of hsa-miR-30a-5p (miR-30a) in OC has not been fully elucidated.

Methods: Three mRNA datasets of normal ovarian tissue and OC, GSE18520,GSE14407 and GSE36668, were downloaded from Gene Expression Omnibus (GEO) to find the differentially expressed gene (DEG). Then the target genes of hsa-miR-30a-5p were predicted by miRWALK3.0 and TargetScan. Then, the gene overlap between DEG and the predicted target genes of miR-30a in OC was analyzed by Gene Ontology (GO) enrichment analysis. Protein-protein interaction (PPI) network was conducted by STRING and Cytoscape, and the effect of HUB gene on the outcome of OC was analyzed.

Results: A common pattern of up-regulation of miR-30a in OC was found. A total of 225 DEG, were identified, both OC-related and miR-30a-related. Many DEG are enriched in the interactions of intracellular matrix tissue, ion binding and biological process regulation. Among the 10 major Hub genes analyzed by PPI, five Hub genes were significantly related to the overall poor survival of OC patients, in which the low expression of ESR1,MAPK10, Tp53 and the high expression of YKT,NSF were related to poor prognosis of OC.
\end{abstract}

Conclusion: Our results indicate that miR-30a is of significance for the biological progress of OC.

Keywords: Differentially expressed genes, Functional factors;miR-30a, Ovarian Cancer

\section{Introduction}

Common gynecological malignant tumors have the following categories: ovarian cancer (OC), cervical cancer, endometrial carcinoma, fallopian tube cancer, vulvar cancer and gestational trophoblastic tumor. Among them, although the incidence of OC is lower than that of cervical cancer and endometrial cancer, the lethal rate has far exceeded both, ranking first in

\footnotetext{
*Correspondence: yuzhiwu_123@126.com

'Weijia Lu and Yunyu Wu contributed equally to this work.

${ }^{4}$ Division of Laboratory Science, Affiliated Cancer Hospital and Institute of Guangzhou Medical University, No.78, Hengzhigang Road, Yuexiu District, Guangzhou 510095, Guangdong, China

Full list of author information is available at the end of the article
}

gynecological malignant tumors. It is reported that more than 200,000 women worldwide suffer from OC, 125000 of whom die from it every year [1]. In the 2018 cancer statistics, 21,530 women in the United States were expected to be diagnosed with OC, and 13,980 died from OC [2]. It is difficult for patients to feel uncomfortable in OC's early stage. And it is often at its advanced stage when patients feel it, accounting for $70 \%$ of all the cases of malignant ovarian tumors [3]. Chirlaque et al. [4] reported that between 2000 and 2004, the 5-year standardized net survival rate of ovarian cancer ranged from $36 \%$ in Spain to $42 \%$ in Belgium, with the net survival

C C The Author(s). 2020 Open Access This article is licensed under a Creative Commons Attribution 4.0 International License, which permits use, sharing, adaptation, distribution and reproduction in any medium or format, as long as you give appropriate credit to the original author(s) and the source, provide a link to the Creative Commons licence, and indicate if changes were made. The images or other third party material in this article are included in the article's Creative Commons licence, unless indicated otherwise in a credit line to the material. If material is not included in the article's Creative Commons licence and your intended use is not permitted by statutory regulation or exceeds the permitted use, you will need to obtain permission directly from the copyright holder. To view a copy of this licence, visit http://creativecommons.org/licenses/by/4.0/ The Creative Commons Public Domain Dedication waiver (http://creativecommons.org/publicdomain/zero/1.0/) applies to the data made available in this article, unless otherwise stated in a credit line to the data. 


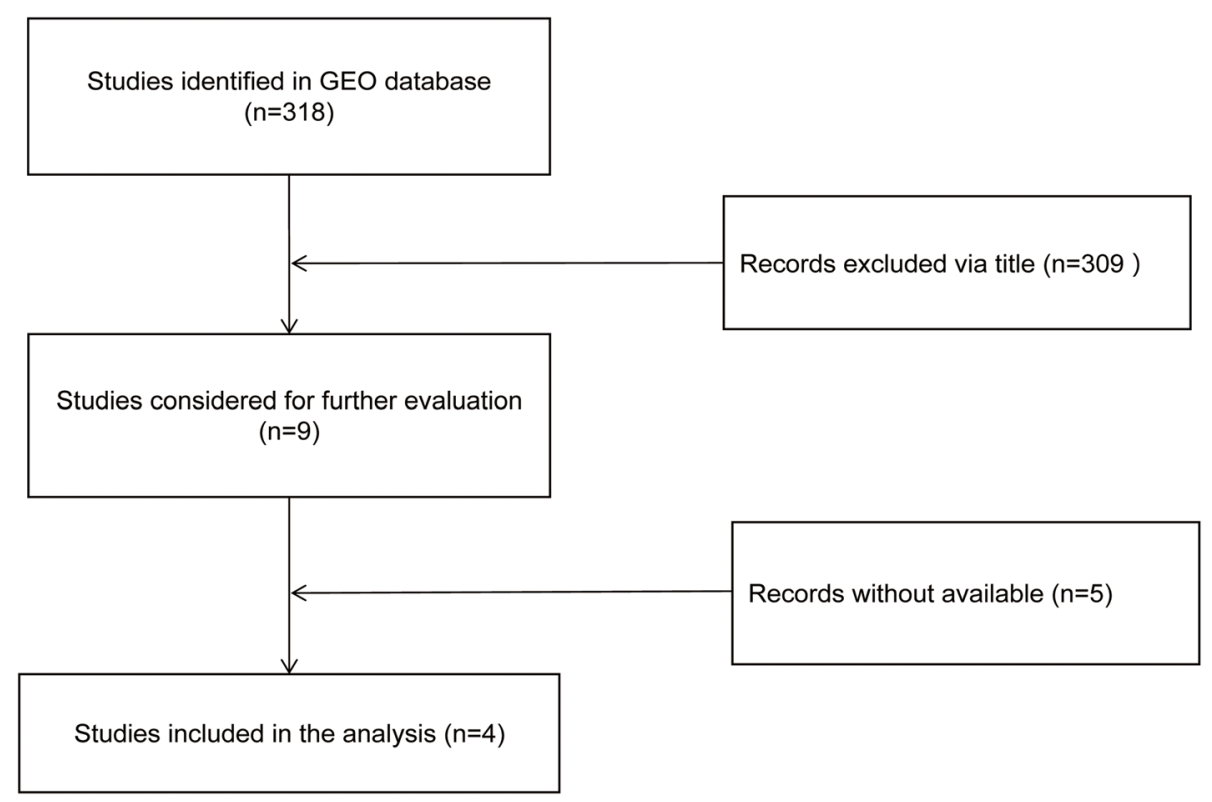

Fig. 1 Research and screening flow Chart of GEO

rate of young people much higher than that of the elderly. Between 1992 and 2004, the net survival rate increased in Belgium, France, Italy, Portugal, Spain and Switzerland, mainly of young and middle-aged women. However, the difference in 5-year net survival rates among these countries in 2004 was greater than that in 1992 [4]. It is obvious that the lethal rate of $\mathrm{OC}$ is high. If we can find a new target relating to the prognosis of OC, clarify its mechanism, and carry out targeted treatment for OC patients, it can not only significantly improve the clinical treatment effect and patients' quality of life, but also play an irreplaceable role in monitoring cancer recurrence and guiding rehabilitation treatment.

MicroRNA (miRNA) is a kind of highly conserved non-coding small molecule RNA. At the posttranscriptional level of mRNA, it regulates gene expression through complete or incomplete complementary pairing with 3 'UTR, CDS or promoter regions of mRNA to inhibit mRNA translation or directly degrade target mRNA [5]. As the miRNA family and its various regulatory functions were discovered, it is explicit that they are widely involved in physiological and pathological processes such as cell differentiation, proliferation and apoptosis, and are closely related to the occurrence and development of a variety of tumors $[6,7]$. More and more studies have shown that the aberrant versions of hsa-miR-30a-5p (miR-30a) are involved in the biological process of a variety of tumors. Some studies have shown that the Lin28b/ IRS1 axis targeting miR-30a-5p promotes the growth of colorectal cancer [8]. Liang et al. [9] believe that plasma miR-30a-5p can be used as a biomarker for early diagnosis and prognosis of lung cancer. Noori et al. [10] reported that microRNA30a and its downstream Snail1 are related to the growth and metastasis of melanoma. The invasive growth associated with miR-30a is mainly mediated by the direct regulation of tumor metastasis and invasion related genes [11]. Besides, studies also found that miR-30a causes ovarian cancer through direct targeting and negative regulation of FOXD1 [12]. MiR-30a expression in ovarian cancer is

Table 1 Basic characteristics of miR-30a related Research on Ovarian Cancer in GEO data set

\begin{tabular}{|c|c|c|c|c|c|c|c|c|}
\hline \multirow[t]{2}{*}{ Study } & \multicolumn{3}{|c|}{ Ovarian cancer tissue } & \multicolumn{3}{|c|}{ Normal ovarian tissue } & \multirow[t]{2}{*}{$t$} & \multirow[t]{2}{*}{$p$} \\
\hline & Mean & SD & $\mathbf{n}$ & Mean & SD & $\mathbf{n}$ & & \\
\hline GSE47841 & 10.33 & 1.62 & 21 & 8.97 & 0.38 & 9 & 3.622 & 0.001 \\
\hline GSE83693 & 8.2 & 1.23 & 16 & 7.47 & 0.92 & 4 & -1.11 & 0.284 \\
\hline GSE53829 & 23 & 1.45 & 45 & 23.44 & 0.95 & 14 & 1.05 & 0.301 \\
\hline GSE23383 & 9.99 & 0.57 & 3 & 8.73 & 1.94 & 3 & 1.08 & 0.343 \\
\hline Total & \multicolumn{8}{|c|}{$\operatorname{SMD}(95 \% \mathrm{Cl})=0.43(-0.33 \sim 1.19), p=0.264,1^{2}=59.9 \%, p=0.058$} \\
\hline
\end{tabular}



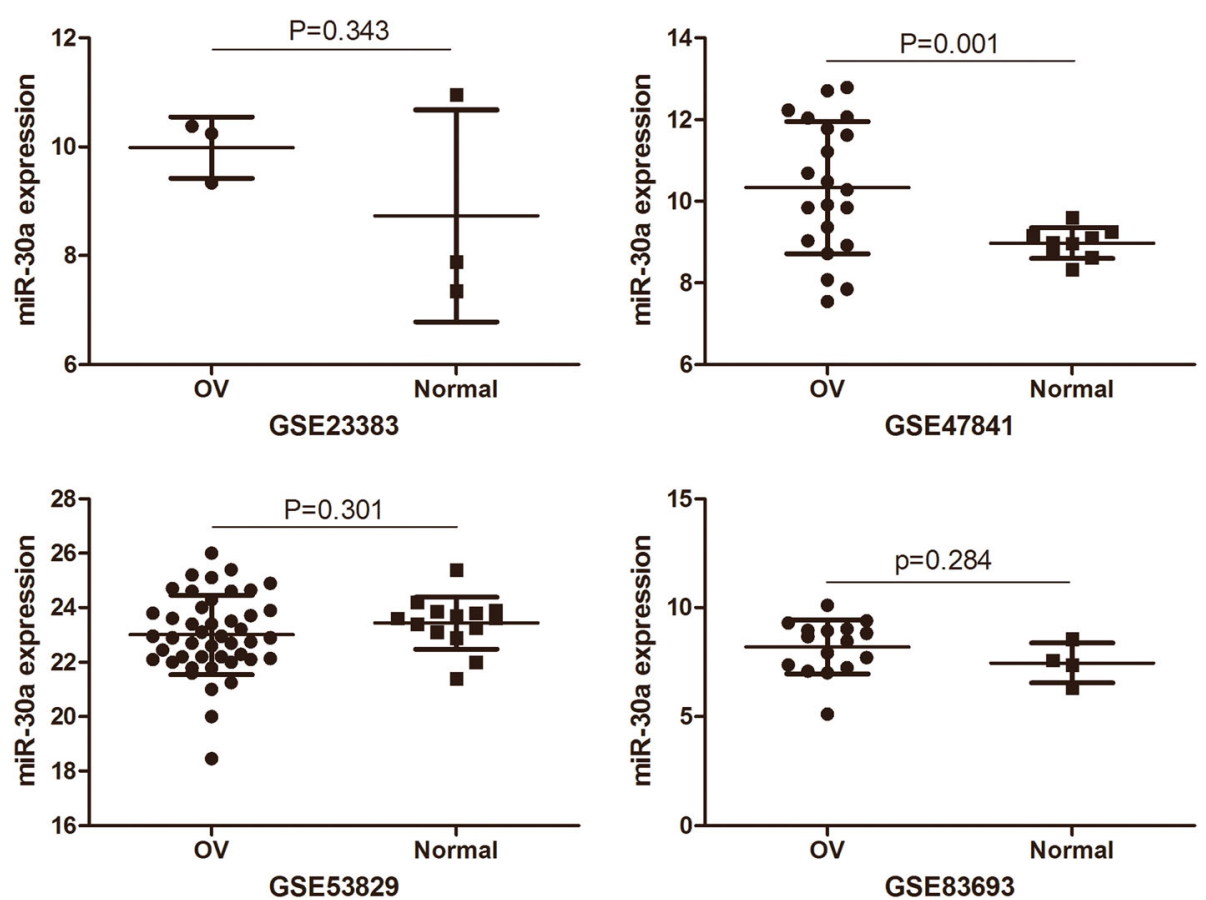

Fig. 2 The expression of ovarian cancer miR-30a was concentrated in GEO data. OV: ovarian cancer tissue; Normal: normal ovarian tissue; miR-30a: hsa-miR-30-5p

significantly up-regulated [12, 13]. Therefore, based on the published data on miR-30a expression in OC,the objective of this study is to identify possible molecular targets and reveal the role of miR-30a by studying miRNAs expression, GEO and study review, and through bioinformatics analysis.

\section{Materials and methods} Selection of GEO dataset

The gene microarray atlas of $\mathrm{OC}$ was obtained from the GEO database (http://www.ncbi.nlm.nih.gov/geo/). The following key words: (ovarian cancer OR ovarian carcinoma OR ovarian) and (microRNA OR non-coding RNA OR miRNA) are used for information retrieval.

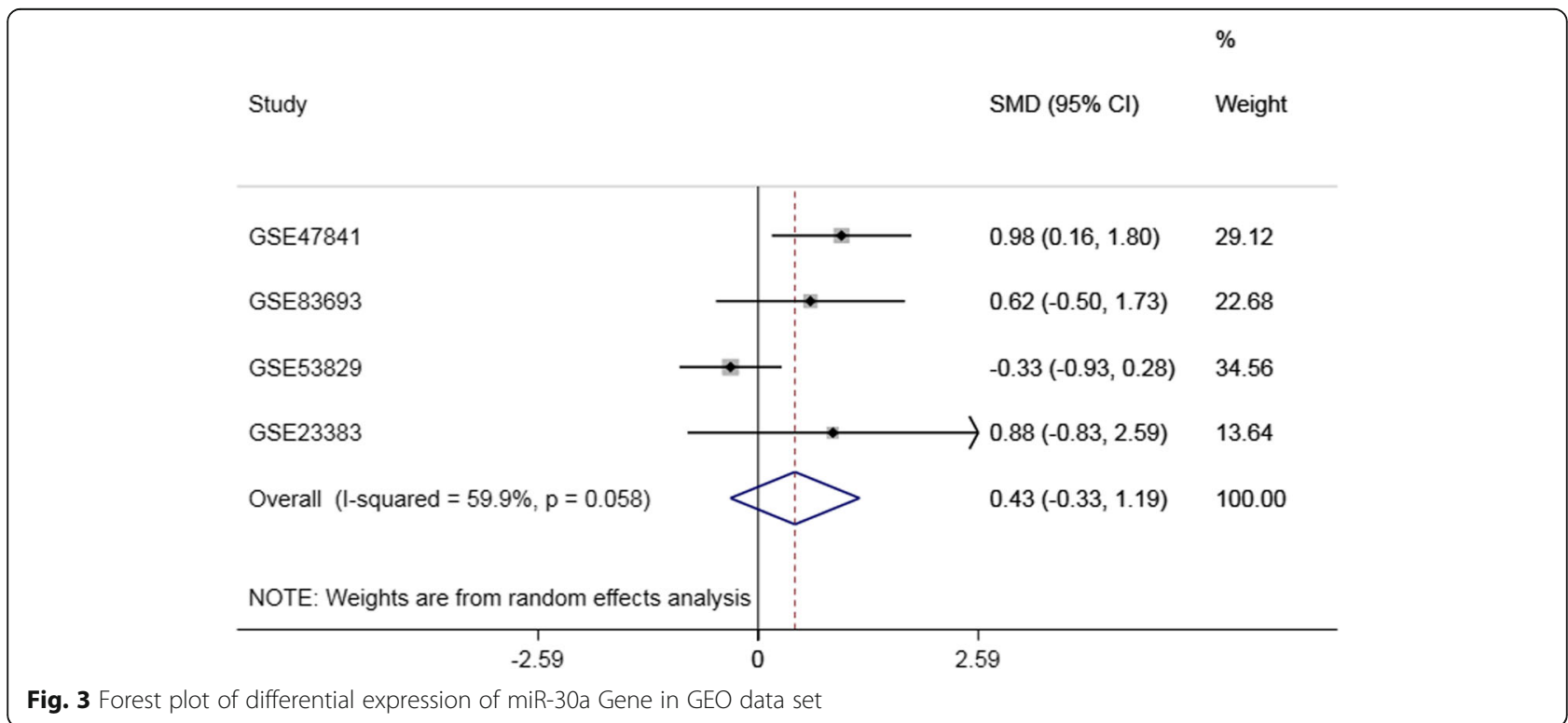




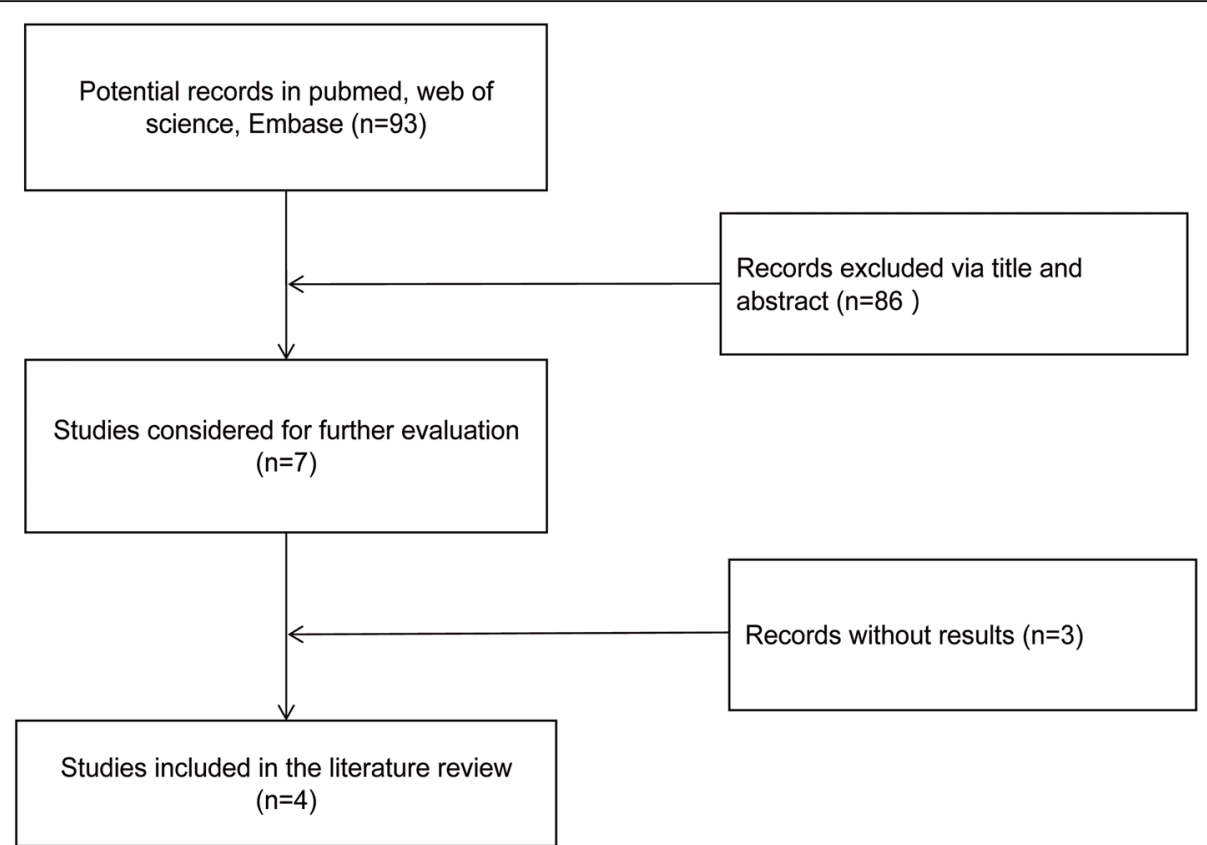

Fig. 4 Screening of literatures on the expression of miR-30a in ovarian cancer

A microarray dataset of miR-30a expression in $\mathrm{OC}$ and normal ovarian tissue (NOT) was included.

\section{Study and data extraction of Mir-30a and ovarian Cancer in the literature}

Through the literature retrieval on PubMed, Web of Science and Embase (till 1st, April, 2020), retrieval words are (microRNA-30 OR miRNA-30 OR miR-30 OR noncoding RNA OR microRNA-30a) AND (ovarian cancer OR ovarian carcinoma OR ovarian tumor). The inclusion and exclusion criteria are as follows: (1) study miR30a expression in OC; (2) exclude reviews, non-clinical studies, case reports, meta-analysis and meeting summaries;(3) exclude those with on control groups.

Gene ontology enrichment and target prediction analysis The gene expression profiles of GSE18520,GSE14407 and GSE36668 come from GEO (http://www.ncbi.nlm. nih.gov/geo). The array data of GSE14407, GSE18520 and GSE36668 are respectively composed of 12, 53 and 4 OC samples; 12, 10 and 4 NOTS samples.

The Limma software package (version 3.6.3) in R/BioManager was conducted to distinguish the DEGs between OC and NOT. By default, the adjusted $P$-value (adj.P.Value) used Benjamini and Hochberg false discovery rate $($ FDR) to correct false-positive results. $P<0.05$ and $|\log 2(\mathrm{FC})|>1$ was set as the cut-off criterion. Based on the platform annotation file downloaded from the database, the probe data in the matrix file was converted into the gene symbols.

\section{Use Online Website}

miRWALK3.0 (https://zmf.umm.uni-heidelberg.de/apps/ zmf/mirwalk2/miRretsys-self.html) and TargetScan (https://www.targetscan.org/vert_72/) to predict the target gene of hsa-miR-30a-5p(TG_miRNA-30a-5p). Then, the gene overlap between the DEG integrated into OC was analyzed by bioinformatics software, and the TG_miRNA$30 \mathrm{a}-5 \mathrm{p}$ was predicted. The Bingo plug-in of Cytoscape

Table 2 Basic characteristics of search literatures related to miR-30a in ovarian cancer

\begin{tabular}{|c|c|c|c|c|c|c|c|c|}
\hline \multirow[t]{2}{*}{ Author } & \multirow[t]{2}{*}{ Year } & \multirow[t]{2}{*}{ Country } & \multicolumn{2}{|l|}{ Case } & \multicolumn{2}{|l|}{ Control } & \multirow[t]{2}{*}{ Result } & \multirow{2}{*}{$\begin{array}{l}\text { Detection } \\
\text { methods }\end{array}$} \\
\hline & & & Source name & $\mathrm{n}$ & Source name & $\mathbf{n}$ & & \\
\hline Wang $Y$ & 2013 & China & low-grade OPSC & 16 & high-grade OPSC & 53 & up-regulation & qRT-PCR \\
\hline Zhou J & 2015 & China & Ovarian cancer urine & 39 & Urine of HP & 30 & up-regulation & qRT-PCR \\
\hline Wang $Y$ & 2018 & China & HGSOC & 11 & NFT & 10 & up-regulation & qRT-PCR \\
\hline Závesky & 2018 & Czech Republic & OCPF & 26 & Blood of HP & 34 & up-regulation & qRT-PCR \\
\hline
\end{tabular}

Note:OPSC: ovarian papillary serous carcinoma;HSSOC:High-grade serous ovarian carcinoma;NFT:normal fallopian tube;OCPF:Ovarian cancer peritoneal fluid;HP:healthy people 

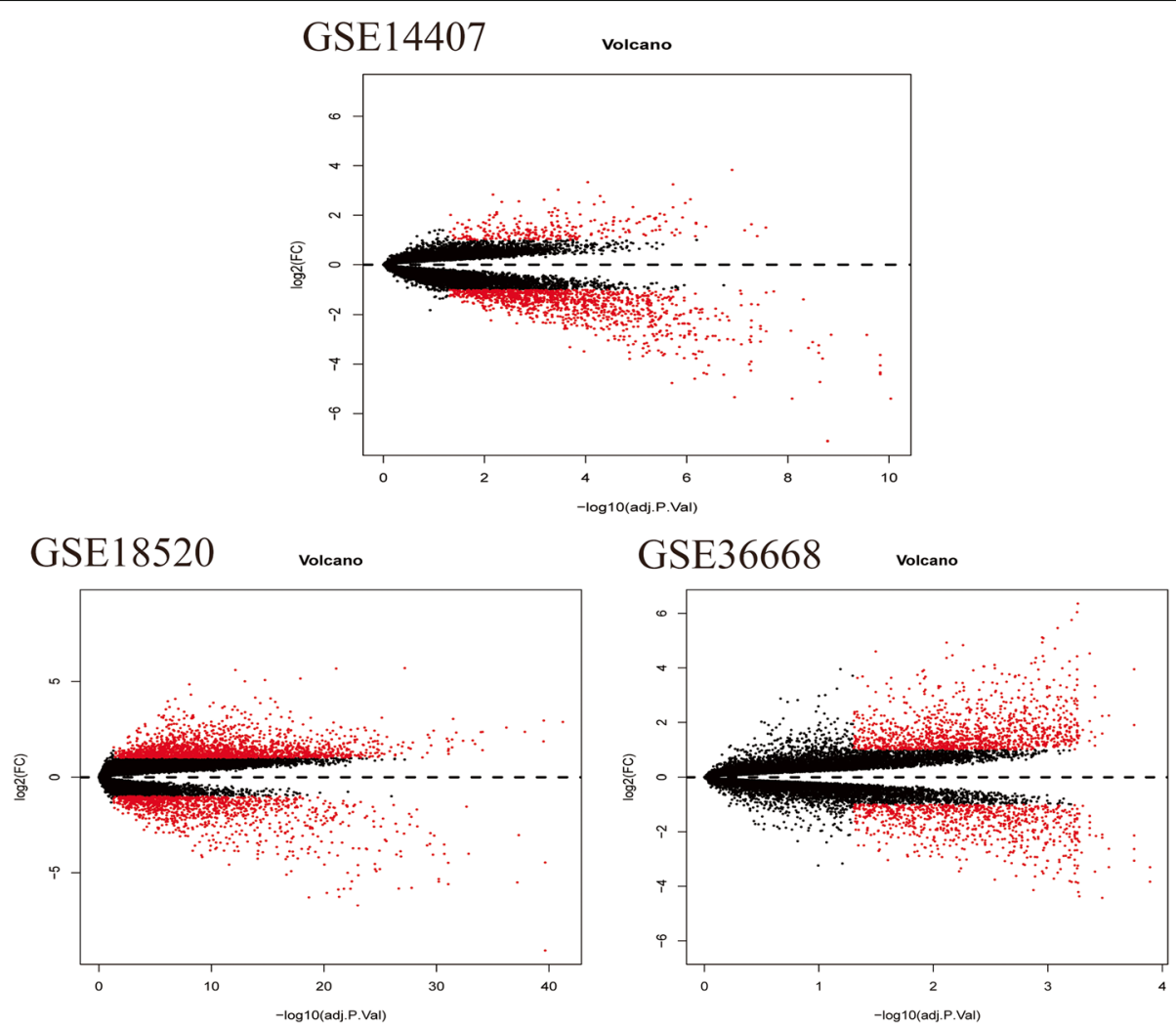

Fig. 5 Volcano Map of Genomewide mRNA Map detected by three Ovarian Cancerrelated data sets from GEO. Aberrantly expressed mRNAs with $P<0.05$ and $\| \log (\mathrm{FC}) \mid>1$ were represented by red. up-regulated genes were indicated by red plots above, down-regulated genes were indicated by red plots belowand nomally expressed mRNAs were indicated by black plots. The x-axis represents the adj.P of-log10. The associated strength is expressed by the $P$ value of each mRNAadj.P.Value, adjusted $P$ value; $F C$, fold change

software (version 3.7.2) was used to analyze the gene overlap by Gene Ontology (GO) and visualize it. The confidence score $\mathrm{C} \geq 0.7$ was set as the truncation standard. Then, the molecular complex (MCODE) was detected. PPI network module of cutoff $=2$, node score cutoff $=0.2$, $\mathrm{k}$-core $=2$ and max.depth $=100$ were screened out.

\section{Survival analysis}

Online tool Kaplan-Meier plotter (KM plotter, www. kmplot.com) can assess the impact of 21 cancers on survival rate. Those that have utmost impacts include the breast (nude 6234), ovarian (nasty 2190), lung (nasty 3452 ), and gastric (nasty 1440) cancer. OC patients were divided into the expression and low expression group, according to the median expression level of specific genes. Kaplan-Meier was used to analyze the overall survival of patients with OC. Calculate and show the hazard ratio (HR) of the 95\% confidence interval (CI).

\section{Statistical analysis}

The data were presented as mean \pm standard deviation (SD). The difference was analyzed by two independent sample t-test between two groups. The correlation between miR-30a level and OC was analyzed by standardized mean difference (SMD) analysis with Stata 15.0 statistical software. Mantel-Haenszel formula (fixed-effects model) or DerSimonia-Laird formula (random-effects model) were used to combine and analyze different GEO data sets. When the Q statistic is significant ( $p \leq 0.05$ or $\mathrm{I}^{2} \geq 50 \%$ ), the random effect model is applied, otherwise the fixed effect model is applied. $P<0.05$ was considered that the difference was statistically significant.

\section{Results}

\section{MiR-30a expression in OC based on GEO}

Based on the GEO dataset (Fig. 1), the expression of miR-30a is accessed in a series of OC and NOT. In this study, four GEO data sets (GSE83693, GSE47841, GSE53829 and GSE23338) were gathered. In the GSE47841 dataset, the miR-30a expression of miR-30 in OC tissue was higher than that in the NOTS $(\mathrm{P}<0.01)$ Compared with NOTs group. While the miR-30 expression of miR-30a in OC tissue increased in the GSE23338 and GSE83693 dataset, and the miR-30a expression of miR-30a in OC tissue decreased in GSE53829 dataset, but all $P$-values were greater than 0.05 . The research 


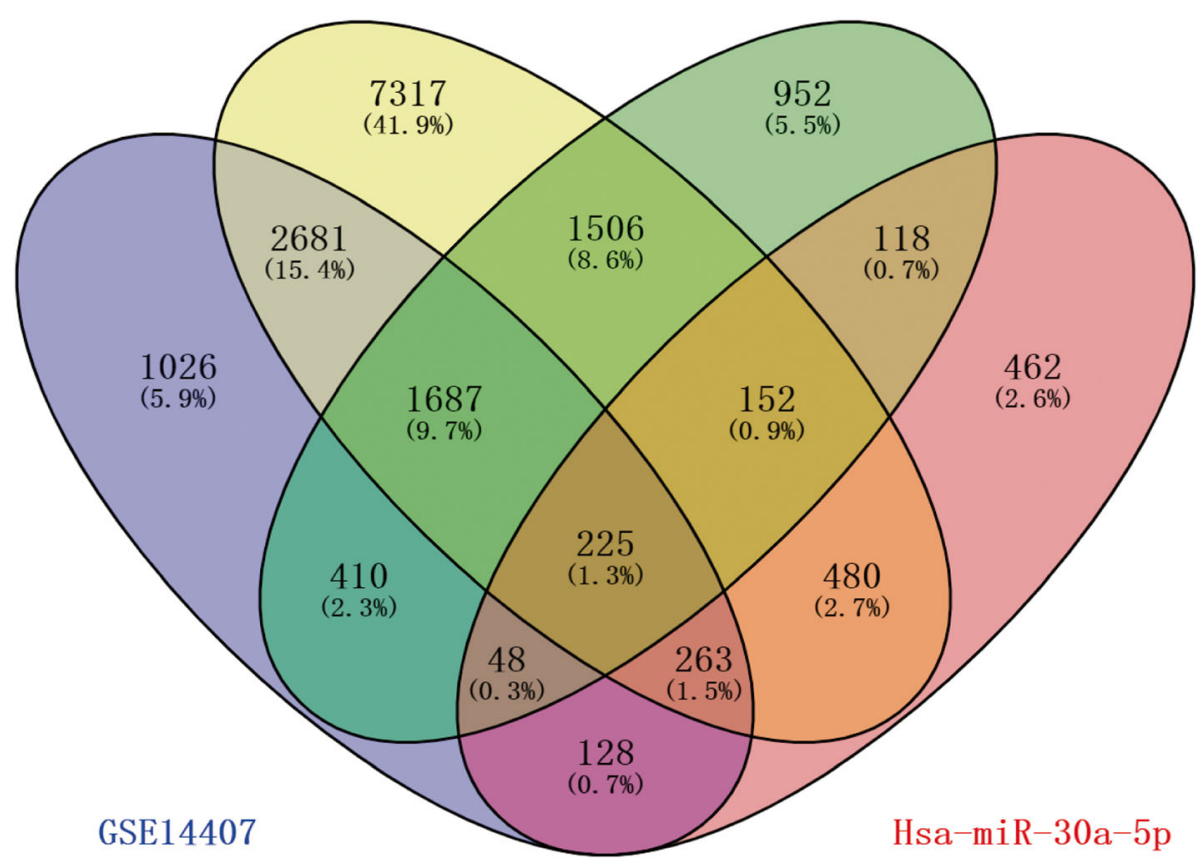

Fig. 6 The Venn map of hsa- mir-30-5p related differentially expressed genes in TG_miR-182-5p and other three GEO data sets, and the overlapping region represents the recognized DEGs. DEGs: differentially expressed genes; TG_miR-30a-5p, target genes of hsa-miRNA-30-5p

features were listed in Table 1 and Fig. 2.However, there was no significant difference between $\mathrm{OC}$ and NOT $(\mathrm{SMD}=0.43,95 \% \mathrm{CI}:-0.33 \sim 1.19, P=0.264)$. The results of the forest plots were shown in Fig. 3.

\section{Expression profile of miR-30a in OC and NOTS}

Then, the expression of miR-30a in ovarian cancer was studied according to the literature data. As shown in Fig. 4, four studies [12-15] up to selection criteria were selected from the literature. Three out of the four studies showed that the expression level of miR-30a in OC tissues was significantly higher than that in non-OC tissues, while in the study, compared with well differentiated ovarian cancer, the level of miR-30a in poorly differentiated ovarian cancer group was significantly higher (Table 2 ).

\section{miR-30a prediction and bioinformatics analysis; data preprocessing and DEGS screening}

6468, 14,311 and 5098 DEG were identified from GSE14407, GSE18520 and GSE36668 datasets respectively. The histological types of ovarian cancer in GSE14407, GSE18520 and GSE36668 data sets were ovarian epithelial cell carcinoma, ovarian epithelial cell carcinoma and ovarian serous carcinoma, respectively.1912 common DEG were filtered out in these three datasets (Figs. 5 and 6) by Venny 2.1.0 (https://bioinfogp.cnb.csic.es/tools/venny/).
Then, 1312 TG_hsa-miR-30a-5p, were predicted based on miRWALK3.0 and TargetScan, of which 225 were verified in 1876 common DEG. According to the gene expression data overview, there are 111 up-regulated hsamiR-30a-5p-related genes and 114down-regulated hsa-miR-30a-5p-related genes in OC tissues compared with non-OC tissues (Fig. 6, Table 3).

\section{Functional analysis of miR-30a-related DEGs in OC}

The functional enrichment analysis is carried out by Bingo plug-in in Cytoscape. The three most important enrichments of molecular function, biological pathway and cell composition were shown in Table 4. The analysis showed that many target genes participated in biological processes such as biological regulation, ion binding and intracellular matrix tissue (Fig. 7). We found that SOX9, transcription factor 21 (TCF21) and Wnt-5a and other genes play a significant role in these key enrichment pathways.

\section{PPI network construction and modules selection}

The PPI network of miR-30a-related DEG consists of 225 nodes and 287 edges, including 111 up-regulated genes and 114 down-regulated genes (Fig. 8). Setting $\geq 10$ degrees as the truncation criteria, 10 genes were selected as Hub genes, and there was a close correlation between HUB genes (Fig. 9a). By using MCODE, an 
Table 3 differentially expressed genes of hsa- mir-30a-5p in ovarian cancer tissues and normal ovarian tissues in GSE18520 dataset (Top 10)

\begin{tabular}{llll}
\hline DEG & \multicolumn{1}{l}{ logFC } & P.Value & adj.P.Val \\
\hline up-regulated genes & & & \\
GLDC & 3.879464906 & $9.08 \mathrm{E}-12$ & $6.62 \mathrm{E}-11$ \\
MAL & 4.080880545 & $4.09 \mathrm{E}-08$ & $1.59 \mathrm{E}-07$ \\
PRSS21 & 2.700693431 & $1.47 \mathrm{E}-11$ & $1.04 \mathrm{E}-10$ \\
GRHL2 & 2.41672613 & $1.49 \mathrm{E}-27$ & $3.11 \mathrm{E}-25$ \\
CTHRC1 & 2.333368517 & $1.48 \mathrm{E}-04$ & $3.27 \mathrm{E}-04$ \\
EYA2 & 2.253746336 & $1.92 \mathrm{E}-05$ & $4.86 \mathrm{E}-05$ \\
SOX9 & 2.077258364 & 0.000000019 & $7.85 \mathrm{E}-08$ \\
E2F7 & 1.961647476 & $5.66 \mathrm{E}-15$ & $7.90 \mathrm{E}-14$ \\
KIAA0101 & 1.916963173 & $4.12 \mathrm{E}-15$ & $5.93 \mathrm{E}-14$ \\
SBK1 & 1.887544551 & $6.62 \mathrm{E}-12$ & $4.95 \mathrm{E}-11$ \\
down-regulated genes & & \\
GFPT2 & -3.731162519 & $4.91 \mathrm{E}-26$ & $7.51 \mathrm{E}-24$ \\
ADAMTS3 & -3.417352741 & $4.99 \mathrm{E}-12$ & $3.83 \mathrm{E}-11$ \\
WNT5A & -3.0965058 & $1.44 \mathrm{E}-08$ & $6.06 \mathrm{E}-08$ \\
HLF & -2.953607331 & $1.97 \mathrm{E}-30$ & $7.46 \mathrm{E}-28$ \\
DPYSL2 & -2.904638189 & $1.50 \mathrm{E}-07$ & $5.35 \mathrm{E}-07$ \\
NR3C2 & -2.607594668 & $7.07 \mathrm{E}-13$ & $6.35 \mathrm{E}-12$ \\
ADRA2A & -2.557257353 & $1.78 \mathrm{E}-13$ & $1.83 \mathrm{E}-12$ \\
TCF21 & -2.488576443 & $2.04 \mathrm{E}-22$ & $1.27 \mathrm{E}-20$ \\
ABI3BP & -2.261098823 & $4.93 \mathrm{E}-20$ & $1.92 \mathrm{E}-18$ \\
POLI & -2.238370149 & $2.41 \mathrm{E}-16$ & $4.43 \mathrm{E}-15$ \\
\hline & & & \\
\hline
\end{tabular}

Table 4 Functional enrichment Analysis of hsa-mir-30a-5p related differentially expressed genes in Ovarian Cancer

\begin{tabular}{llll}
\hline Term & Description & Count & FDR \\
\hline Biological processes & & \\
GO:0009987 & cellular process & 192 & 0.003 \\
GO:0065007 & biological regulation & 169 & 0.00017 \\
GO:0050789 & regulation of biological process & 163 & 0.00016 \\
Molecular Function (Go) & & \\
GO:0005488 & binding & 175 & $2.91 \mathrm{E}-05$ \\
GO:0043167 & ion binding & 99 & 0.0018 \\
GO:0097159 & organic cyclic compound binding & 91 & 0.0017 \\
Cellular Component & & \\
GO:0005622 & intracellular & 190 & 0.0033 \\
GO:0044424 & intracellular part & 189 & 0.0031 \\
GO:0043226 & organelle & 174 & 0.0031 \\
\hline
\end{tabular}

important module was obtained from the PPI network of miR-30a-related DEG, including 7 nodes and 17 edges (Fig. 9b).

\section{Survival analysis}

The prognostic value of 10 HUB genes in the PPI network was accessed on the www.kmplot.com website. According to the high and low expression of each HUB gene,we analyzed the overall survival of patients with OC. The results showed that low expression of ESR1 (HR $=0.84,95 \% \mathrm{CI}: 0.74 \sim 0.96, P=0.01)$, low expression of MAPK10 (HR = 0.77,95\%CI:0.60 0.97,P = 0.03), low expression of $\mathrm{Tp} 53 \quad(\mathrm{HR}=0.84,95 \% \mathrm{CI}: 0.73 \sim 0.98, P=$ 0.023), high expression of YKT6 (high expression of $\mathrm{HR}=1.16,95 \% \mathrm{CI}: 1.02 \sim 1.33, P=0.029)$, and high expression of NSF $(\mathrm{HR}=1.23,95 \% \mathrm{CI}: 1.07 \sim 1.40, P=0.003)$ were associated with overall survival decreasing of patients with ovarian cancer (Fig. 10).

\section{Discussion}

In this study, based on the data of GEO dataset and published studies, we identified the aberrant expression of miRNA related to $\mathrm{OC}$ by comparing the expression profiles of miRNA in OC and NOTS tissues. In addition, through GO analysis, PPI network and Kaplan-Meier plotter, the new markers and potential targets of miR-30a took part in the regulation of key biological processes of $\mathrm{OC}$ were identified and analyzed.

By far, few researches have been done on the characteristics of miR-30a in OC. In 2014, the result of Zhou et al. [13] showed the level of miR-30a expression in urine in $\mathrm{OC}$ was higher than that of healthy controls.

Some studies showed that miR-30a expression was up-regulated in the tissues of ovarian cancer [12, 15]. Interestingly, the study of, Wang et al. [14] showed that the level of miR-30a expression in poorly differentiated ovarian papillary serous carcinoma tissues was obviously higher than that in well-differentiated ovarian papillary serous carcinoma tissues. According to the GEO dataset, only the GSE47841 dataset showed an increase in miR-30a expression in OC, while other datasets showed no statistical difference. As the expression level of miR-30a in OC is still controversial, further studies are needed to clarify the role of $\mathrm{miR}-30 \mathrm{a}$ in $\mathrm{OC}$.

MiR-30a is one of the quite important miRNAs in OC regulation. Lee et al. [16] suggested that miR-30a expression was noteworthily increased in $\mathrm{OC}$, and the high level of miR-30a expression was related to the significant shortening of disease-free survival. In addition, Gong et al. [16] found that TMED2 is an oncogene and a potential target for the treatment of epithelial ovarian cancer, while TMED2 increases the 


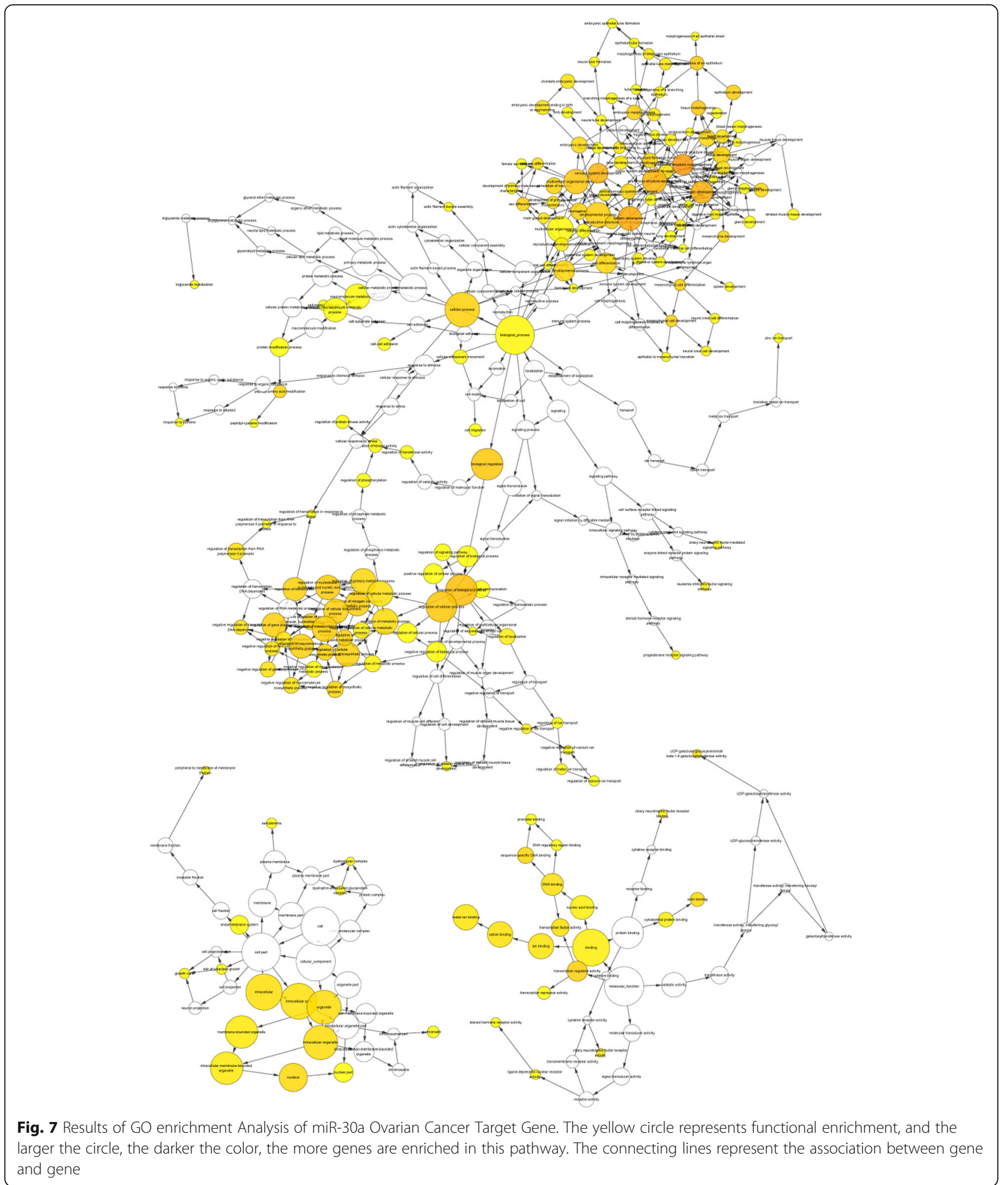

expression of IGF1R by competing with miR-30a. The study of Han et al. [17] showed that the feedback loop between miR-30a-5p and DNMT1 mediated the resistance of ovarian cancer cells to cisplatin. Liu et al. [18] found that high expression of miRNA-30a-5p can promote cell growth and colony formation, and enhance cell migration and invasion. Therefore, miRNA-30a-5p is expected to become a significant new target for drug resistance treatment of ovarian cancer. Given the current 


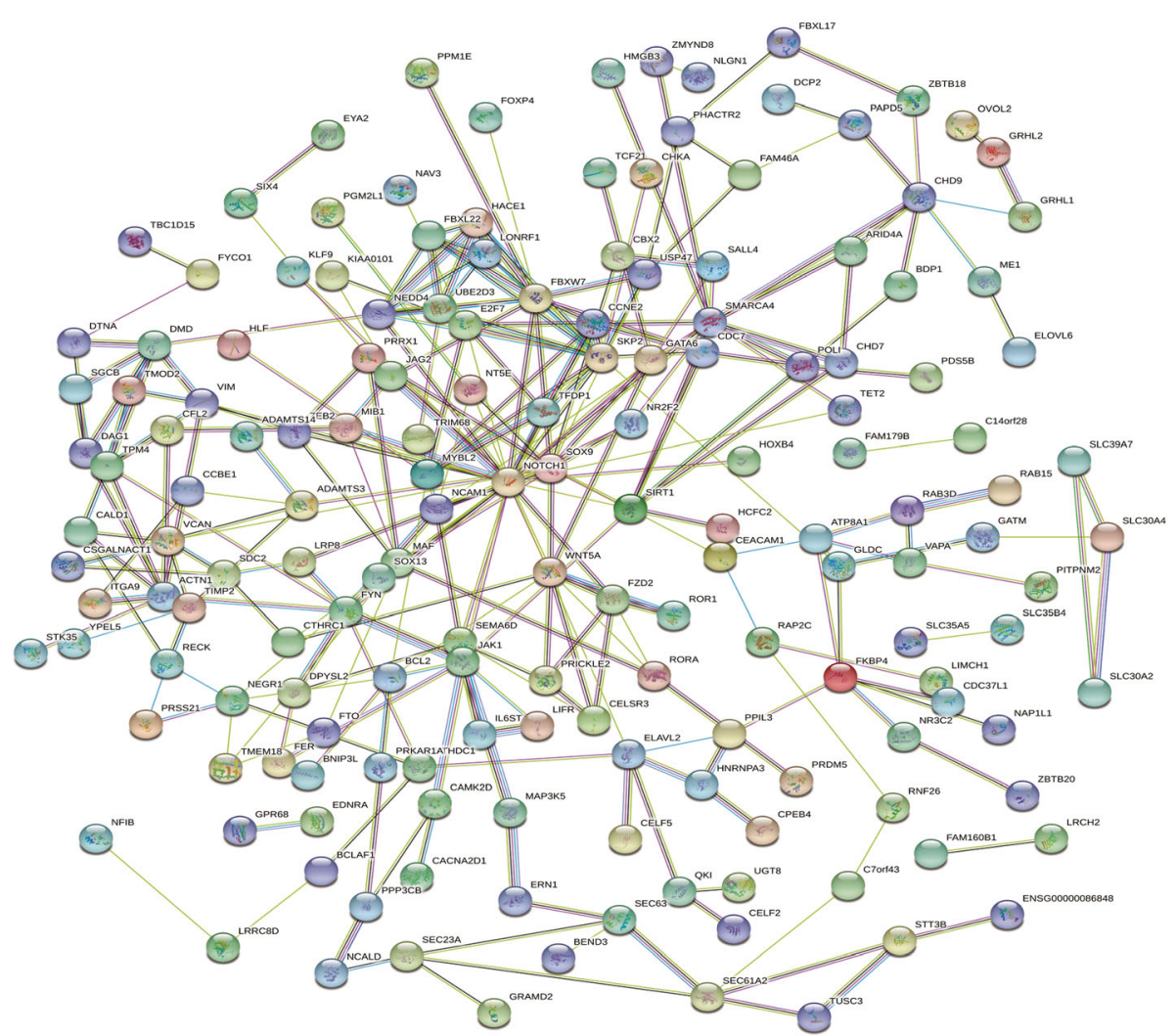

Fig. 8 Protein-protein interaction network of hsa-miR-30a-5p-related DEGs. The lines represent interaction relationship between nodes. DEGs, differentially expressed genes. The line between the circle nodes represents the interaction between the two proteins linked by the line. Colored nodes:query proteins and first shell of interactors;white nodes:second shell of interactors;empty nodes:proteins of unknown 3D structure;illed nodes:some 3D structure is known or predicted.

situation, it is necessary to further clarify the molecular mechanism and clinical importance relating to the aberrant expression of miR-30a in ovarian cancer.

ESR1 encodes the estrogen receptor, a ligandactivated transcription factor, which is composed of the binding of several important hormones, DNA binding and the important domain of transcriptional activation. Estrogen and its receptors play an important role in hypoplasia and reproductive function, and are also involved in pathological processes including breast cancer, endometrial cancer and osteoporosis [19]. MAPK10, also known as JNK3, belongs to the subgroup of JUN N-terminal kinase (JNK) in the mitogen-activated protein kinase (MAPK) family. The study of Silvia et al. [20] showed that the prognosis of ovarian cancer patients with low expression of ESR1 was poor compared with that of high expression. This is consistent with the conclusion of this study. Dai et al. [21] reported that the expression of MAPK10 protein was negatively correlated with the overall survival time of OC. It is not consistent with the conclusion of this study. In this study, the survival analysis of Hub gene relating to miR-30a target gene showed that the expression of MAPK10 protein was low and the overall survival time of OC was significantly shortened. MAPK10 is inclined to be considered as a tumor suppressor gene in current studies [22], and more studies are needed to verify it. The Tp53 gene encodes a tumor suppressor protein that includes transcriptional activation, DNA binding and oligomerization domains. The coding protein regulates the expression of target genes in response to a variety of cellular stress, thereby inducing cell cycle arrest, apoptosis, senescence, DNA repair or metabolic changes [23]. Hurley et al. [24] reported that the sensitivity and specificity of the TP53 and PAX8 joint detection of ovarian cancer were 56 and $98 \%$, respectively. The study of Chen et al. [25] showed that ubiquitin ligase TRIM71 inhibits the occurrence of ovarian tumors by degrading mutant In this study, the survival analysis of Hub gene relating to miR-30a target gene showed that the low expression of $\mathrm{Tp}$ protein was closely related to the poor prognosis of 

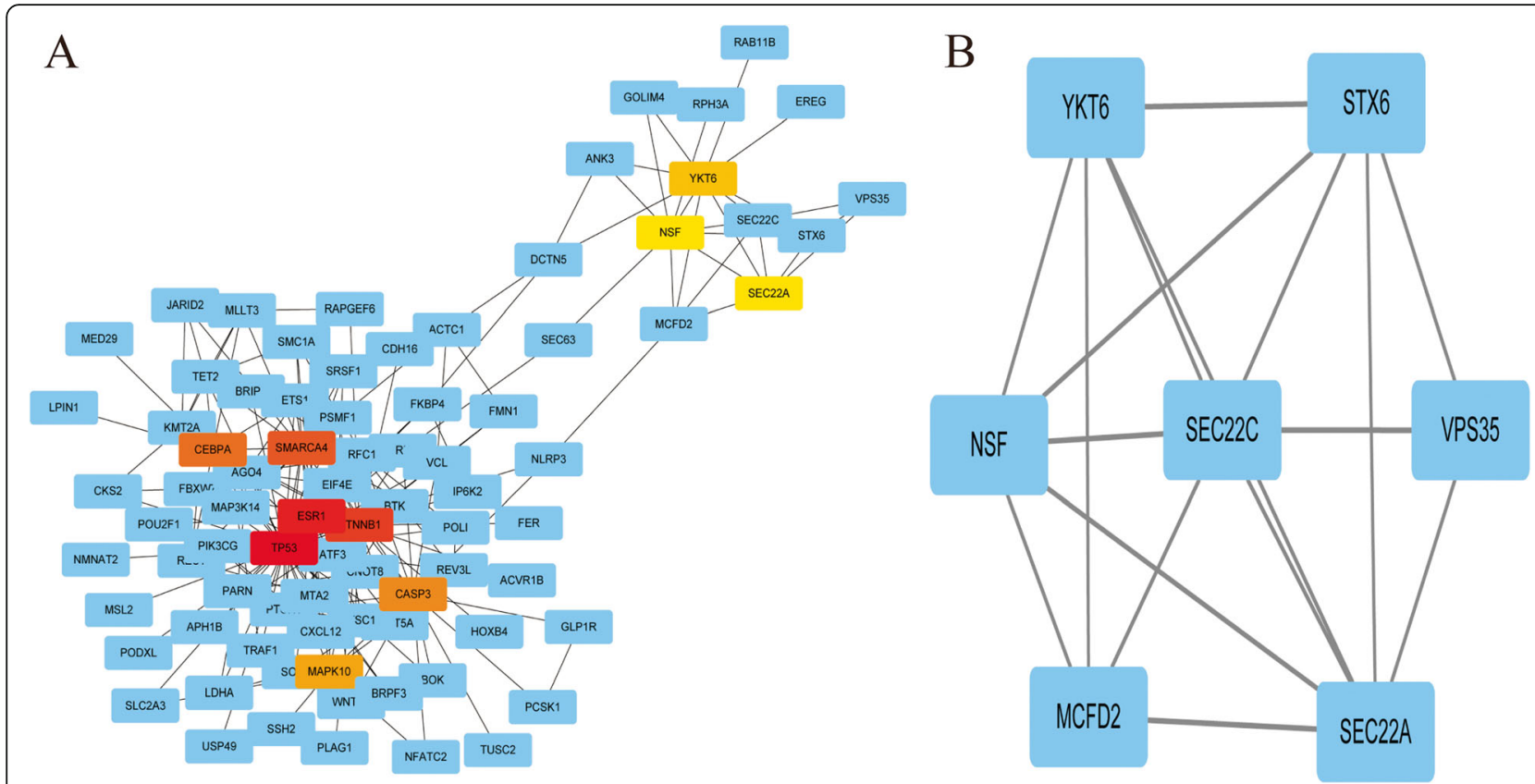

Fig. 9 Protein-protein interaction network. a: Protein-protein interaction network of hube genes of hsa-miR-30a-5p-related DEGs. b: A significant module selected from protein-protein interaction network of hsa-miR-30a-5p-related DEGs. Red and yellow represent the key Hub genes of Tp10. The darker the color, the stronger the association with other genes in the PPI network. The lines represent interaction relationship between nodes. DEGs,differentially expressed genes

OC. YKT6, also known as synaptic vesicle protein homologue YKT6 precursor, is a protein encoded by YKT6 gene. Marc et al. [26] reported that patients with non-small cell lung cancer with high expression of YKT6 protein had shorter both diseasefree survival and overall survival. In this study, the survival analysis of Hub gene relating to miR-30a target gene showed when YKT6 protein was highly expressed, the prognosis of OC patients was poor.

N-ethylmaleimide-sensitive factor (NSF) is an oligomeric protein with a molecular weight of $76 \mathrm{KDa}$, an ATP enzyme involved in membrane fusion [27]. Lee et al. [28] studies have shown that the overall survival rate of colorectal cancer patients with nuclear dominant expression of GS28 (Golgi snare protein, $28 \mathrm{kDa}$ ), a member of the NSF attachment protein receptor family, is significantly lower than that of patients with non-nuclear dominant expression. The results of this study showed when the expression of NSF was high, the prognosis of patients with OC was poor. Thus, YKT6, NSF is closely related to $\mathrm{OC}$ and is expected to become a new prognostic marker of OC.

In the current study, we found that miR-30amediated DEGs are involved in the regulation of key biological processes of ovarian cancer, such as SOX9, transcription factor 21 (TCF21), Wnt-5a,etc. Transcription factor Sox9 belongs to the HMG protein family and is related to early ovarian development [29]. Previous studies have shown that SOX9 plays a needful role in tumorigenesis and metastasis. Siu et al. [30] reported that the migration and invasion of ovarian cancer cells were regulated by hexokinase 2 through FAK/ERK1/2/MMP9/ NANOG/SOX9 signal pathway. Raspaglio et al. [31] found that SOX9 expression is up-regulated in OC and is related to the poor prognosis of the controls. Our current study shows that the level of SOX9 mRNA expression in $\mathrm{OC}$ is higher than that in NOTS. We assume that SOX9 is a cancerpromoting factor. TCF21 is a recently discovered new tumor suppressor factor, which is widely expressed in interstitial cells or their derived tissue cells during the development of cardiovascular system, genitourinary system and respiratory system, and has a great influence on cell growth and differentiation [32]. Duan et al. and others [33] found that TCF21 can target PI3K/Akt and ERK signal pathways, thus inhibiting tumor-associated angiogenesis and cholangiocarcinoma growth. Many pieces of evidence show that TCF21 is regulated by miRNAs, such as miR526b [34], miR-205 [35], which can inhibit tumor development. Zhou et al. [36] found that microRNA-30-3p targeting TCF21 inhibited endothelial cell injury induced by inflammatory factors. Based on the above, we speculate 


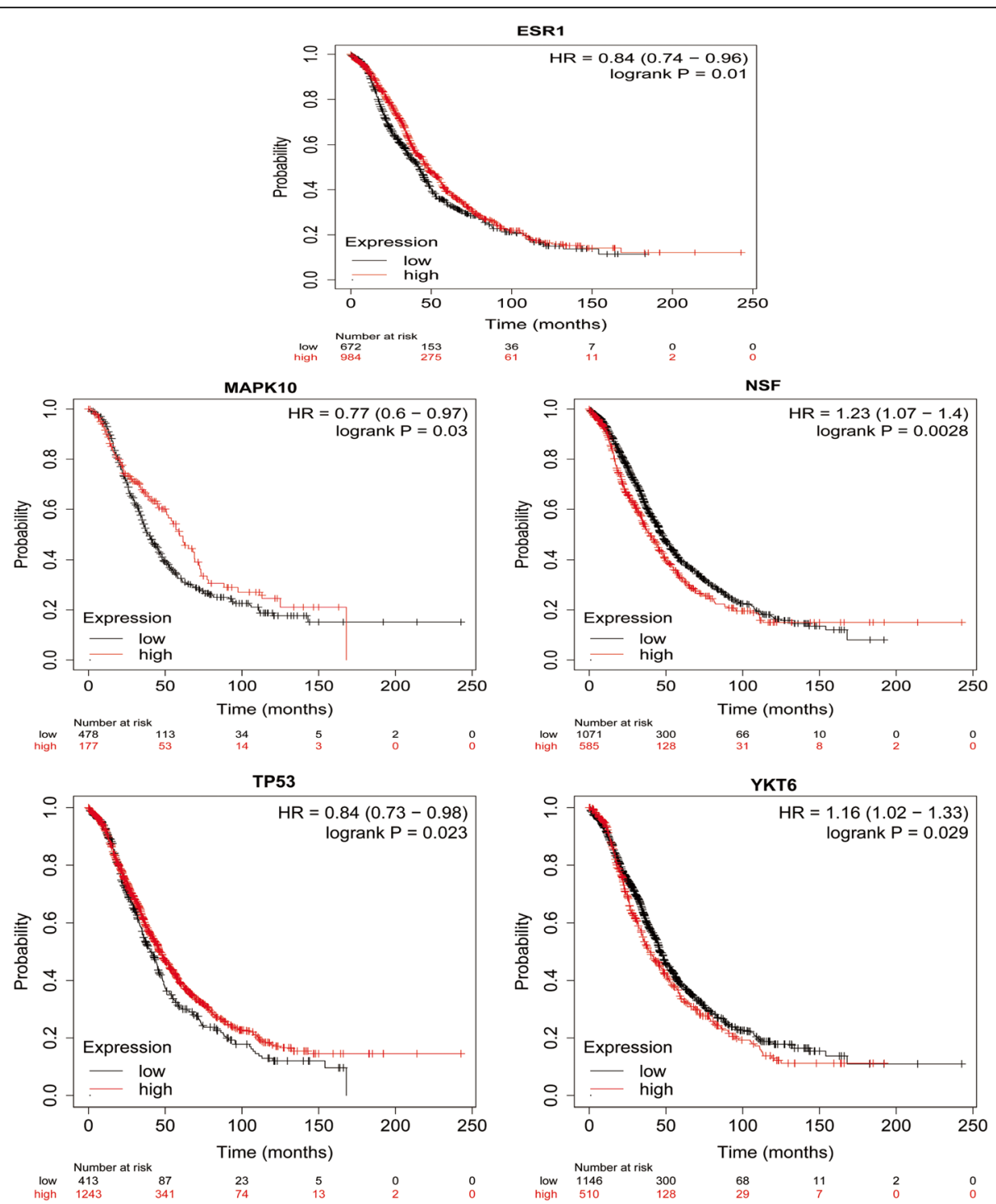

Fig. 10 The expression of ESR1, MAPK10, Tp53, YKT, NSF and the prognosis of patients with ovarian cancer were analyzed. According to the median expression level, patients with ovarian cancer were divided into high expression group and low expression group

that TCF21 may be the target gene of miR-30a in OC. Wnt ligands belong to a family of at least 19 secretory proteins, which play a critical role in cell differentiation, proliferation and histogenesis. Wnt$5 \mathrm{a}$ is one of the quite important factors in the nonclassical pathway of Wnt signaling, which can activate messy proteins through tuberculosis of receptors on the cell membrane, and thus promote proliferation like cell polarity establishment and cytoskeleton rearrangement [37]. Arabzadeh et al. [38] reported that in human ovarian cancer cell line SKOV-3, compared with normal controls, the expression of WNT5A was significantly decreased and had immunomodulatory activity. It is consistent with our current research.
In conclusion, Our study indicated that miR-30a is of importance in OC biology. However, further in vivo and in vitro researches are needed to study its pathogenesis in order to verify the key role of molecular networks regulated by miR-30a in OC.

\section{Abbreviations}

MiRNAs: MicroRNAs; miR-30a: Has-miR-30a-5p; GEO: Gene Expression Omnibus; OC: Ovarian cancer; DEG: Differentially expressed gene; GO: Gene Ontology; FDR: False discovery rate; SMD: Standardized mean difference; HR: Hazard ratio; NOT: Normal ovarian tissue; JNK: JUN N-terminal kinase; MAPK: Mitogen-activated protein kinase; NSF: N-ethylmaleimide-sensitive factor; TCF21: Transcription factor 21

\section{Acknowledgments}

Our paper is supported by the National Natural Science Foundation of China (General Program;Key Program;Major Program). Grant No.81902991. 


\section{Authors' contributions}

Weijia Lu, MD, Yunyu Wu, Zhiwu Yu: Critical revision of the manuscript; Weijia Lu,MD,Yunyu Wu,Zhiwu Yu: Substantial contribution to the conception and design of the work, manuscript drafting;Weijia Lu,CanXiong Lu,Ting Zhu,ZhongLu Ren: Acquisition, analysis, and interpretation of the data; Weijia Lu,MD,Yunyu Wu,Zhiwu Yu: Revising the manuscript critically, final approval of the version to be published. All authors have read and approved the final article.

\section{Availability of data and materials}

The datasets used and/or analysed during the current study are available from the corresponding author on reasonable request.

\section{Ethics approval and consent to participate}

Ethical approval was not needed because this is a Bioinformatics analysis.

\section{Consent for publication}

Not applicable.

\section{Competing interests}

All the authors declare that they have no conflict of interest.

\section{Author details}

${ }^{1}$ Guangzhou University of Chinese Medicine, No.232, Waihuandong Road, University Town, Panyu District, Guangzhou 510006, Guangdong, China. 2Department of Gynaecological Oncology, Affiliated Cancer Hospital and Institute of Guangzhou Medical University, Guangzhou 510095, Guangdong Province, China. ${ }^{3}$ Laboratory Department, Foshan Sanshui hospital of Traditional Chinese Medicine, Foshan 528100, Guangdong, China. ${ }^{4}$ Division of Laboratory Science, Affiliated Cancer Hospital and Institute of Guangzhou Medical University, No.78, Hengzhigang Road, Yuexiu District, Guangzhou 510095, Guangdong, China. ${ }^{5}$ College of Medical Information Engineering Guangdong Pharmaceutical University, Guangzhou 510006, Guangdong, China.

\section{Received: 16 June 2020 Accepted: 18 September 2020}

\section{Published online: 01 October 2020}

\section{References}

1. Sankaranarayanan R, Ferlay J. Worldwide burden of gynaecological cancer: The size of the problem. Best Pract Res Clin Obstet Gynaecology. 2006;20(2):207-25.

2. Siegel RL, M KD, J A. Cancer statistics, 2018. Ca A Cancer Journal for Clinicians. 2018;68(1):7.

3. Cortez AJ, Tudrej P, Kujawa KA, Lisowska KM. Advances in ovarian cancer therapy. Cancer Chemother Pharmacol. 2017:81:17-38.

4. Chirlaque MD, Uhry Z, Salmerón D, Sánchez-Zapata Ml, Zannoni GF, Navarro C. Trends in net survival from ovarian cancer in six European Latin countries: results from the SUDCAN population-based study. Eur J Cancer Prev. 2017:S107-13.

5. Lujambio A, Lowe SW. The microcosmos of cancer. NATURE. 2012;482(7385): 347-55.

6. Palma CA, Al Sheikha D, Lim TK, Bryant A, Vu TT, Jayaswal V, et al. MicroRNA-155 as an inducer of apoptosis and cell differentiation in acute myeloid Leukaemia. Mol Cancer. 2014;13:79.

7. Raghavachari N, Liu P, Barb JJ, Yang Y, Wang R, Nguyen QT, et al. Integrated analysis of miRNA and mRNA during differentiation of human CD34+ cells delineates the regulatory roles of microRNA in hematopoiesis. Exp Hematol. 2014;42(1):14-27.

8. Tang M, Zhou J, You L, Cui Z, Zhang H. LIN28B/IRS1 axis is targeted by miR30a-5p and promotes tumor growth in colorectal cancer. J Cell Biochem. 2019.

9. Liang LB, Zhu WJ, Chen XM, Luo FM. Plasma miR-30a-5p as an early novel noninvasive diagnostic and prognostic biomarker for lung cancer. Future oncology (London, England). 2019:15(32):3711-21.

10. Noori J, Haghjooy Javanmard S, Sharifi M. The role of microRNA-30a and downstream snail1 on the growth and metastasis of melanoma tumor. Iran Basic Med Sci. 2019;22(5):534-40.

11. Wang L, Zhao S, Yu M. Mechanism of low expression of miR-30a-5p on epithelial-Mesenchymal transition and metastasis in ovarian Cancer. DNA Cell Biol. 2019;38(4):341-51

12. Wang Y, Qiu C, Lu N, Liu Z, Jin C, Sun C, et al. FOXD1 is targeted by miR$30 a-5 p$ and miR-200a-5p and suppresses the proliferation of human ovarian carcinoma cells by promoting p21 expression in a p53-independent manner. Int J Oncol. 2018;52(6):2130-42.
13. Zhou J, Gong G, Tan H, Dai F, Wen J. Urinary microRNA-30a-5p is a potential biomarker for ovarian serous adenocarcinoma. Oncol Rep. 2015:33(6):2915-23.

14. Wang Y, Li L, Qu Z, Li R, Bi T, Jiang J, et al. The expression of miR-30a* and miR-30 $e^{*}$ is associated with a dualistic model for grading ovarian papillary serious carcinoma. Int J Oncol. 2014;44(6):1904-14.

15. Závesky L, Jandáková E, Weinberger V, Miná L, Hanzíková V, Du Ková D, et al. Ascites-derived extracellular microRNAs as potential biomarkers for ovarian Cancer. Reprod Sci. 2018;26(4):1-13.

16. Lee H, Park CS, Deftereos G, M V, Stern JE, Hawes SE, et al. MicroRNA expression in ovarian carcinoma and its correlation with clinicopathological features. World J Surg Oncol. 2012;10:174.

17. Han X, Zhen S, Ye Z, Lu J, Wang L, Li P, et al. A feedback loop between miR-30a/c-5p and DNMT1 mediates Cisplatin resistance in ovarian Cancer cells. Cell Physiol Biochem. 2017;41(3):973-86.

18. Liu J, Wu X, Liu H, Liang Y, Gao X, Cai Z, et al. Expression of microRNA-30a$5 p$ in drug-resistant and drug-sensitive ovarian cancer cell lines. Oncol Lett. 2016:12(3):2065-70.

19. Pinkerton JV, Conner EA. Beyond estrogen: advances in tissue selective estrogen complexes and selective estrogen receptor modulators. Climacteric. 2019;22(2):140-7.

20. Darb-Esfahani S, Wirtz RM, Sinn BV, Budczies J, Noske A, Weichert W, et al. Estrogen receptor $1 \mathrm{mRNA}$ is a prognostic factor in ovarian carcinoma: determination by kinetic PCR in formalin-fixed paraffin-embedded tissue. Endocr Relat Cancer. 2009;16(4):1229-39.

21. Dai $F$, Zhang $Y$, Chen $Y$. Involvement of miR-29b signaling in the sensitivity to chemotherapy in patients with ovarian carcinoma. Hum Pathol. 2014;45(6):1285-93.

22. Qiao B, Wang Q, Zhao Y, Wu J. miR-205-3p Functions as a Tumor Suppressor in Ovarian Carcinoma. Reproductive sciences (Thousand Oaks, Calif.). 2020;27(1):380-8.

23. Uxa S, Bernhart SH, Mages CFS, Fischer M, Kohler R, Hoffmann S, et al. DREA $\mathrm{M}$ and $\mathrm{RB}$ cooperate to induce gene repression and cell-cycle arrest in response to p53 activation. Nucleic Acids Res. 2019:47(17):9087-103.

24. Hurley LC, Levin NK, Chatterjee M, Coles J, Muszkat S, Howarth Z, et al. Evaluation of paraneoplastic antigens reveals TRIM21 autoantibodies as biomarker for early detection of ovarian cancer in combination with autoantibodies to NY-ESO-1 and TP53. Cancer biomarkers : section A of Disease markers. 2020;27(3):407-21

25. Chen $Y$, Hao Q, Wang J, Li J, Huang $C$, Zhang $Y$, et al. Ubiquitin ligase TRIM71 suppresses ovarian tumorigenesis by degrading mutant p53. Cell Death Dis. 2019;10(10):737.

26. Ruiz-Martinez M, Navarro A, Marrades RM, Viñolas N, Santasusagna S, Muñoz $C$, et al. YKT6 expression, exosome release, and survival in non-small cell ung cancer. Oncotarget. 2016;7(32):51515-24.

27. Kawasaki F, Ordway RW. Molecular mechanisms determining conserved properties of short-term synaptic depression revealed in NSF and SNAP-25 conditional mutants. P NATL ACAD SCI USA. 2009;106(34):14658-63.

28. Lee $\mathrm{SH}$, Yoo HJ, Rim DE, Cui Y, Lee A, Jung ES, et al. Nuclear expression of GS28 protein: a novel biomarker that predicts prognosis in colorectal cancers. Int J Med Sci. 2017:14(6):515-22.

29. Rimm EB, Giovannucci EL, Stampfer MJ, Colditz GA, Litin LB, Willett WC. Reproducibility and validity of an expanded self-administered Semiquantitative food frequency questionnaire among male health professionals. Am J Epidemiol. 1992:10:10.

30. Siu MKY, Jiang YX, Wang JJ, Leung THY, Han CY, Tsang BK, et al. Hexokinase 2 Regulates Ovarian Cancer Cell Migration, Invasion and Stemness via FAK ERK1/2/MMP9/NANOG/SOX9 Signaling Cascades. Cancers. 2019;11:6.

31. Raspaglio G, Petrillo M, Martinelli E, Li Puma DD, Mariani M, De Donato M, et al. Sox9 and Hif-2a regulate TUBB3 gene expression and affect ovarian cancer aggressiveness. GENE. 2014;542(2):173-81.

32. Li Q, Lei Y, Du W. A novel target of p53, TCF21, can respond to hypoxia by MAPK pathway inactivation in uterine Corpus endometrial carcinoma. DNA Cell Biol. 2018;37(5):473-80.

33. Duan HX, Li BW, Zhuang X, Wang LT, Cao Q, Tan LH, et al. TCF21 inhibits tumor-associated angiogenesis and suppresses the growth of cholangiocarcinoma by targeting PI3K/Akt and ERK signaling. American journal of physiology. Gastrointestinal Liver Physiology. 2019;316(6):G763-73.

34. Shin B, Feser R, Nault B, Hunter S, Maiti S, Ugwuagbo KC, et al. miR526b and miR655 Induce Oxidative Stress in Breast Cancer. INT J MOL SCI. 2019:20:16.

35. Wei J, Zhang L, Li J, Zhu S, Tai M, Mason CW, et al. MicroRNA-205 promotes cell invasion by repressing TCF21 in human ovarian cancer. J Ovarian Res. 2017;10(1):33 
36. Zhou Z, Chen Y, Zhang D, Wu S, Liu T, Cai G, et al. MicroRNA-30-3p suppresses inflammatory factor-induced endothelial cell injury by targeting TCF21. Mediat Inflamm. 2019;2019:1342190.

37. Kuss P, Kraft K, Stumm J, Ibrahim D, Vallecillo-Garcia P, Mundlos S, et al. Regulation of cell polarity in the cartilage growth plate and perichondrium of metacarpal elements by HOXD13 and WNT5A. Dev Biol. 2014;385(1):83-93.

38. Arabzadeh S, Hossein G, Zarnani AH. Wnt5A exerts immunomodulatory activity in the human ovarian cancer cell line SKOV-3. Cell Biol Int. 2016; 40(2):177-87.

\section{Publisher's Note}

Springer Nature remains neutral with regard to jurisdictional claims in published maps and institutional affiliations.

Ready to submit your research? Choose BMC and benefit from:

- fast, convenient online submission

- thorough peer review by experienced researchers in your field

- rapid publication on acceptance

- support for research data, including large and complex data types

- gold Open Access which fosters wider collaboration and increased citations

- maximum visibility for your research: over $100 \mathrm{M}$ website views per year

At $B M C$, research is always in progress. 